\title{
Identification of Endothelial-to-Mesenchymal Transition as a Potential Participant in Radiation Proctitis
}

Elodie Mintet, ${ }^{*}$ Emilie Rannou, ${ }^{*}$ Valérie Buard, * Gail West, ${ }^{\dagger}$ Olivier Guipaud, * Georges Tarlet, ${ }^{*}$ Jean-Christophe Sabourin, Marc Benderitter, ${ }^{\S}$ Claudio Fiocchi, ${ }^{\dagger}$ Fabien Milliat, ${ }^{*}$ and Agnès François*

From the Department of Radiobiology and Epidemiology, * Radiobiology and Radiopathology Research Laboratory, and the Department of Radiobiology and Epidemiology, ${ }^{\S}$ Institut de Radioprotection et de Sûreté Nucléaire, Fontenay-aux-Roses, France; the Department of Pathobiology, ${ }^{\dagger}$ Digestive Disease Institute, Lerner Research Institute, Cleveland Clinic Foundation, Cleveland, Ohio; and the Department of Pathology, ${ }^{\ddagger}$ Rouen University Hospital, Rouen, France

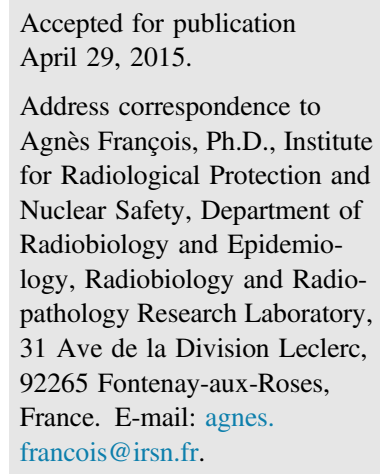

\begin{abstract}
The endothelial-to-mesenchymal transition (EndoMT) is a crucial cellular process during heart development necessary to the formation of cardiac valves. This embryonic process reappears in several pathological situations, such as vascular injury or organ fibrosis of various etiologies, as a mediator of extracellular matrix-producing cells. Because radiation induces both vascular damage and fibrosis, we investigated whether radiation exposure induces EndoMT in primary human intestinal microvascular endothelial cells (HIMECS) and whether EndoMT contributes to radiation-induced rectal damage in humans and in a preclinical model of radiation proctitis in mice. Irradiated HIMECs show phenotypic hallmarks of radiationinduced endothelial cell activation in vitro. Moreover, HIMECs undergo changes in molecular expression pattern compatible with EndoMT, with up-regulation of mesenchymal markers and down-regulation of endothelial markers via transforming growth factor/Smad pathway activation. In vivo, EndoMT readily occurs in the human rectum after radiation therapy for rectal adenocarcinoma. Finally, EndoMT was observed in rectal mucosal and submucosal microvessels in a preclinical model of radiation proctitis in Tie2-green fluorescent protein reporter-expressing mice all along radiation proctitis development, also associated with transforming growth factor/Smad pathway activation. In conclusion, radiation-induced cell activation and tissue inflammation constitute a setting that fosters the phenotypic conversion of endothelial cells into mesenchymal cells. Therefore, EndoMT is identified as a potential participant in radiation-induced gut damage and may represent an interesting therapeutic target in cases of radiation-induced pelvic disease. (Am J Pathol 2015, 185: 2550-2562; http://dx.doi.org/10.1016/j.ajpath.2015.04.028)
\end{abstract}

Endothelial cells display a plastic behavior and undergo what is referred to as the endothelial-to-mesenchymal transition (EndoMT). ${ }^{1}$ Similar to the epithelial-to mesenchymal transition, EndoMT is characterized by the progressive phenotypic transition of endothelial cells to a fibroblast/ myofibroblast or smooth muscle cell-like phenotype. EndoMT is reflected by the loss of specific endothelial cell markers (vascular endothelial cadherin and platelet endothelial cell adhesion molecule-1) and the de novo expression of several mesenchymal cell markers, such as $\alpha$-smooth muscle actin ( $\alpha$-SMA), a marker of myofibroblastic activation, or smooth muscle $22 \alpha$, which is specifically expressed by smooth muscle cells, together with the loss of cell-cell junctions and the acquisition of invasive and migratory properties. EndoMT is a common physiological process occurring during embryogenesis, such as cardiac development. ${ }^{2,3}$ In adult tissues, EndoMT occurs in pathological conditions, such as cardiac, renal, or pulmonary fibrosis. ${ }^{3-5}$ A recent study identified endothelium as the origin, via EndoMT, of neointimal hyperplasia responsible for graft failure after coronary bypass surgery in humans. ${ }^{6}$ EndoMT is also responsible for a significant fraction of ectopic ossifying

Supported by the Institute for Radiological Protection and Nuclear Safety ROSIRIS Program.

Disclosures: None declared. 
lesions in cases of fibrodysplasia ossificans progressiva, a genetic disorder characterized by ectopic bone formation and ossification of soft tissues. ${ }^{7}$ Finally, EndoMT participates in tumor progression as an inducer of carcinomaassociated fibroblasts. $^{8}$

Radiation proctitis is a common and debilitating consequence of radiation therapy for pelvic tumors, such as rectal and prostatic carcinoma, and radiation-induced gastrointestinal toxicity remains a limiting factor in the application of radiation therapy. Radiation-induced damage to the gastrointestinal tissue is characterized by acute inflammation, followed by progressive tissue scarring leading to organ fibrosis. ${ }^{9,10}$ Vascular abnormalities in irradiated tissue have been observed in patients undergoing radiotherapy for cancer, and endothelial injury has been described as a crucial event in the initiation and progression of tissue damage. ${ }^{10,11}$ Long-term endothelial cell activation induced by irradiation favors the maintenance of a procoagulant phenotype and immune cell recruitment, both of which may participate in chronic fibrosis. ${ }^{12}$ Tissue fibrosis is characterized by sustained production and deposition of extracellular matrix by activated mesenchymal cells, primarily myofibroblasts, resulting in tissue remodeling, which may compromise organ function. ${ }^{13}$ The origins of myofibroblasts in tissue fibrosis are multiple. ${ }^{14}$ Resident mesenchymal cells, such as fibroblasts or smooth muscle cells, respond to the inflammatory insult by switching to a myofibroblastic phenotype, acquiring $\alpha$-SMA-positive stress fibers and secreting fibrillar collagens; hematopoietic mesenchymal progenitors may be recruited from the bloodstream ${ }^{15}$; and cells of nonmesenchymal origin, such as epithelial and endothelial cells, may transform into mesenchymal or smooth muscle-like cells and participate in organ fibrosis. ${ }^{16,17}$ Although EndoMT has been demonstrated in several organs, such as the injured heart, lung, and kidney, less is known concerning the gut, and especially radiation-induced damage to the gastrointestinal tract. A first study demonstrated an epithelial-to-mesenchymal transition in the pathogenesis of fistulae in human Crohn disease, and more recently an epithelial-to-mesenchymal transition was identified in a preclinical model of trinitrobenzene sulfonic acid-induced intestinal fibrosis in mice. ${ }^{18,19}$ Finally, Rieder et $\mathrm{al}^{20}$ detected, for the first time, EndoMT in inflamed human intestinal mucosa and in a preclinical model of colonic fibrosis in mice. EndoMT is governed by several signaling pathways, among which include transforming growth factor (TGF)- $\beta 1$ and its canonical Smad2/3 signaling cascade, and TGF- $\beta 2$ signaling through the Smad, mitogen-activated protein kinase/extracellular-signal regulated kinase, phosphatidylinositol 3-kinase, and P38 mitogenactivated protein kinase pathways. ${ }^{6,21-23}$ TGF- $\beta$ is, by the way, used as a potent inducer of EndoMT both in vitro and in vivo. ${ }^{4,8} \mathrm{We}$ previously published that radiation-induced vascular damage in human radiation proctitis and irradiated endothelial cells in vitro show overexpression of TGF- $\beta$ and phospho-Smad2/3, conditions that may favor EndoMT. ${ }^{24,25}$

Given the fact that EndoMT has never been reported in irradiated gut and considering that intestinal fibrosis of various etiologies may share common mechanisms, we assumed that EndoMT could participate in the development of radiation-induced intestinal fibrosis.

Herein, we show that irradiated human intestinal microvascular endothelial cells (HIMECs) acquire a mesenchymal-like phenotype suggestive of radiation-induced EndoMT in vitro via TGF/Smad pathway activation. EndoMT also occurs in human radiation proctitis. In vivo, there is evidence of EndoMT in the rectal mucosal and submucosal vessels using a preclinical model of radiation proctitis in Tie2-green fluorescent protein (GFP) reporter-expressing mice, from day 7 to 14 weeks after irradiation, associated also with TGF/Smad pathway activation. These data suggest that radiation-induced damage to the healthy rectum may cause phenotypic conversion of endothelial cells into a mesenchymal-like phenotype, which may participate in tissue injury, and reinforce the key role of the microvasculature in the development of radiation damage to gastrointestinal tissue.

\section{Materials and Methods}

\section{Cell Culture}

HIMECs were isolated from surgically resected specimens of normal human colon, as previously described. ${ }^{26}$ Cells were grown in EBM-2MV (Lonza, Verviers, Belgium) and subcultured in 6-well plates. Confluent HIMEC monolayers were exposed to $0,2,10$, or $20 \mathrm{~Gy} \gamma$ irradiation with a ${ }^{137} \mathrm{Cs}$ source (IBL 637; CisBio, Saclay, France; dose rate, 1 Gy/ minute). Normal intestinal myofibroblasts (Lonza) were cultured in SmGM-2 medium (Lonza).

For immunofluorescence, cells were incubated with polyclonal rabbit anti-human von Willebrand factor (vWF; Dako, Les Ulis, France), mouse monoclonal anti-human $\alpha$-SMA clone 1A4 (Dako), goat polyclonal anti-human smooth muscle 22- $\alpha$ (Abcam, Paris, France), or rabbit polyclonal anti-phospho-Smad2/3 (Ser423/Ser425; Santa Cruz Biotechnology, Heidelberg, Germany), followed by corresponding secondary antibodies (Alexa Fluor-conjugated antibodies; Life Technologies, Saint Aubin, France). For double immunostaining, primary antibodies were incubated together, and corresponding secondary antibodies too. Slides were mounted in Vectashield mounting medium with DAPI (Eurobio/Abcys, Courtaboeuf, France).

$\beta$-Catenin/phalloidin co-immunostaining: cells were incubated with primary rabbit monoclonal anti- $\beta$-catenin antibody (Abcam), followed by corresponding secondary antibody (Alexa Fluor-conjugated antibody; Life Technologies). A CytoPainter F-actin staining kit, red fluorescence (Abcam), was used, according to the manufacturer's instructions, to stain phalloidin. Slides were mounted in Vectashield mounting medium without DAPI (Eurobio/Abcys).

\section{Human Rectal Tissue}

Human rectal tissue was obtained at the Department of Pathology, Gustave Roussy Institute (Villejuif, France), following institutional ethical guidelines and French Medical Research 
A
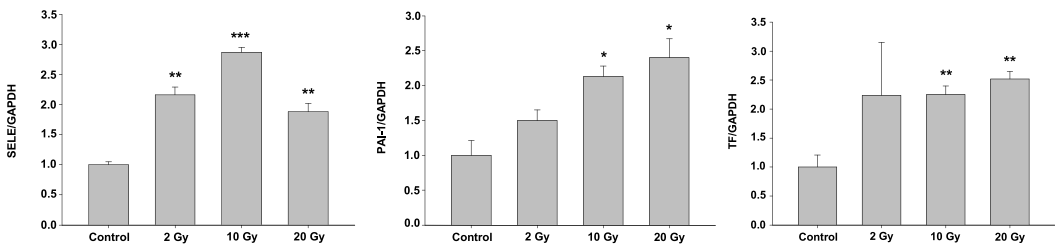

B
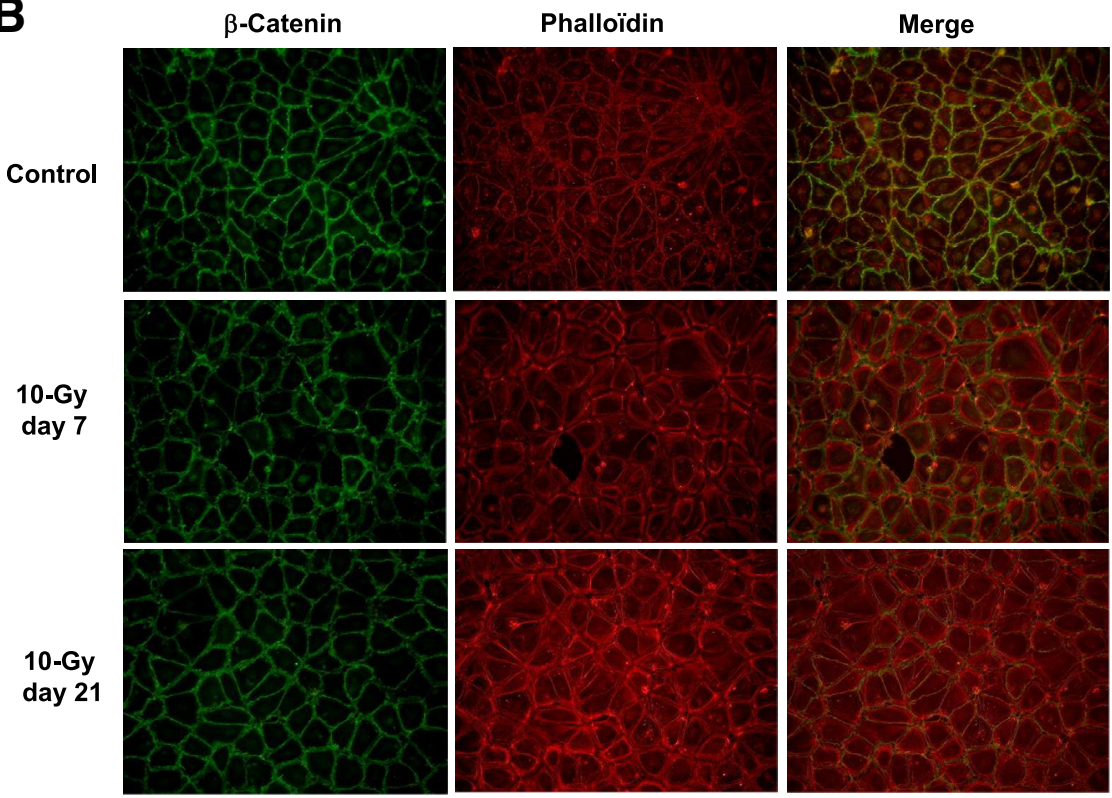

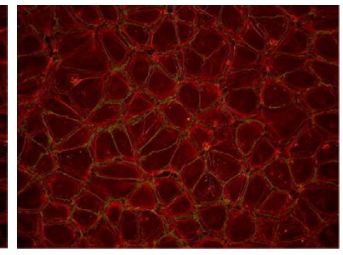

Figure 1 Human intestinal microvascular endothelial cells (HIMECs) are activated by ionizing radiation. A: Real-time quantitative PCR analyses of HIMEC expression of E-selectin (SELE), plasminogen activator inhibitor-type 1 (PAI-1), and tissue factor (TF) 7 days after $0-, 2-, 10-$, or 20-Gy radiation exposure, showing classic radiation-induced endothelial cell activation. B: Double-immunofluorescence staining of control and 10-Gy-irradiated HIMECs 7 and 21 days after exposure with human anti- $\beta$-catenin antibody (green) and F-actin cytopainting kit using phalloidin conjugate (red). Merged images are presented in the right column. Radiation exposure induces a global increase in cellular stress fibers. Data are representative of three independent experiments performed in triplicate $(\mathbf{A})$. Data are presented as means $\pm \operatorname{SEM}(\mathbf{A}) .{ }^{*} P<0.05$, ${ }^{*} P<0.01$, and ${ }^{* * *} P<0.001$. Original magnification, $\times 200$ (B). GAPDH, glyceraldehyde-3phosphate dehydrogenase.
Council guidelines. Tissue specimens from 24 patients treated for rectal adenocarcinoma with preoperative radiotherapy (45 Gy; fractions of 1.8 or $2 \mathrm{~Gy}$ ) were included. Tumors were surgically resected 5 to 7 weeks after treatment. For each patient, specimens of normal tissue were taken adjacent to the tumor (in the irradiation field) and distant from the tumor (outside the irradiation field), making each patient his or her own control. ${ }^{25}$ Paraffin-embedded rectal sections ( $5 \mu \mathrm{m}$ thick) were stained with hematoxylin/eosin/saffron for routine examination. Radiation injury scores were determined independently by two authors (A.F. and F.M.), and discrepancies were resolved in conference. Briefly, individual abnormalities in each tissue compartment were assessed as normal or abnormal, ranked according to severity, as described in Supplemental Table S1. The sum of the scores for each parameter in all compartments (the retrieved sum to 100) constituted the radiation injury score.

For immunofluorescence double labeling, sections were incubated with monoclonal mouse anti-human smooth muscle $\alpha$, clone 1A4 (Dako), and polyclonal rabbit antihuman vWF (Dako) at the same time, followed by corresponding secondary Alexa Fluor-conjugated antibodies (Life Technologies). Sections were mounted in Vectashield mounting medium with DAPI (Eurobio/Abcys).

\section{Animal Model and Irradiation}

The protocol (P13 to P18) for animal use in this project was reviewed and approved by the national ethics committee 81 .
Tg(TIE2GFP)287Sato/J mice (TIE2-GFP mice) expressing the green fluorescent protein under the control of endothelial promoter Tie2 were from the Jackson Laboratory (Bar Harbor, ME). Animals were anesthetized (1.5\% isoflurane), and a single dose of $27-\mathrm{Gy} \gamma$ irradiation $(1.4 \mathrm{~Gy} /$ minute) was delivered by a cobalt 60 source through a $1 \times 0.8-\mathrm{cm}$ window centered on the colorectal region. This model of localized single-dose radiation exposure does not simulate fractionation treatment, but generates histopathological lesions similar to those seen clinically (ie, severe acute mucosal ulceration and transmural collagen deposition during the late phase), with $100 \%$ rectal obstruction, as previously reported. ${ }^{27-29}$

At different times after radiation exposure, colorectal tissues were removed and paraffin sections $(5 \mu \mathrm{m}$ thick) were stained with hematoxylin-eosin-saffron for routine histological examination.

\section{GFP Immunostaining}

Sections were incubated with goat polyclonal to GFP primary antibody (Abcam), followed by a biotinylated rabbit anti-goat IgG secondary antibody (Dako), and counterstained with Mayer's hematoxylin.

\section{Immunofluorescence}

Sections were incubated with a rabbit anti- $\alpha$-SMA primary antibody (Abcam), followed by an Alexa Fluor 488-conjugated 


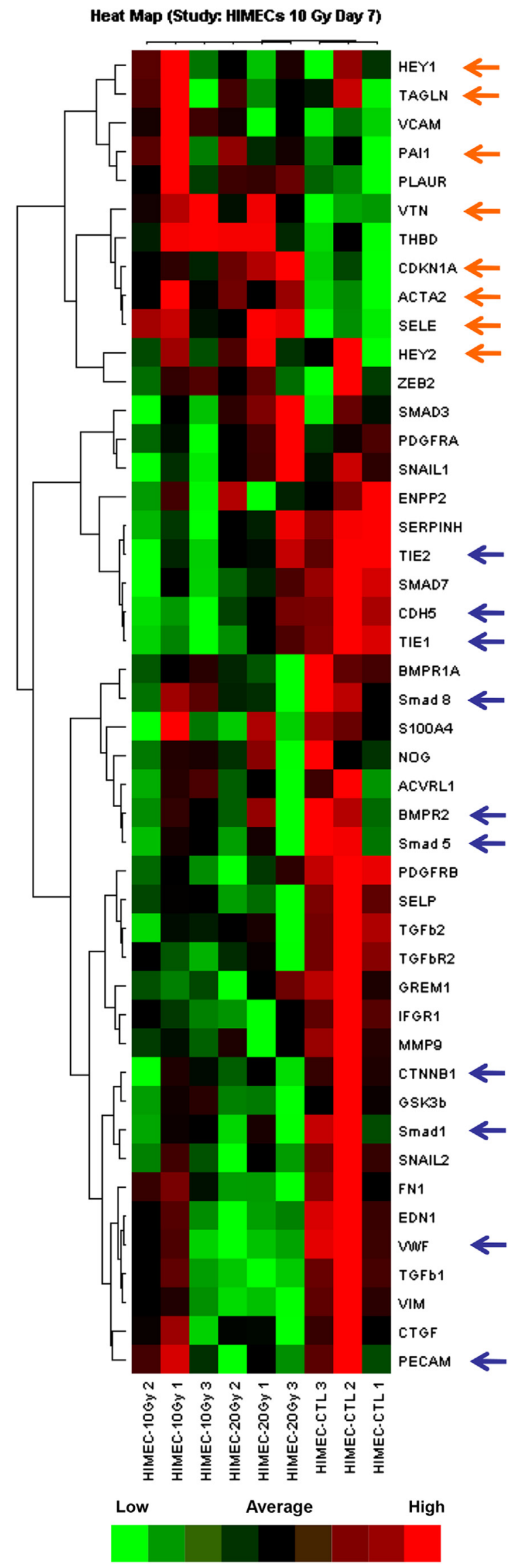

goat anti-mouse antibody (Life Technologies). For GFP and $\alpha$ SMA or phospho-Smad2/3 double-immunofluorescence staining, sections were incubated with rabbit polyclonal antibody to $\alpha$-SMA (Abcam) or to phospho-Smad2/3 (Santa Cruz Biotechnology), followed by Alexa Fluor 568-conjugated goat anti-rabbit antibody, and with goat polyclonal antibody to GFP/ fluorescein isothiocyanate (Abcam). Sections were mounted in ProLong Gold Antifade Mountant with DAPI (Life Technologies). Confocal analyses were performed on a Zeiss LSM 780 confocal imaging system (Zeiss France, Marly le Roi, France).

\section{Chimeric Mice}

TIE2-GFP mice were prepared with 9.5 Gy total body irradiation, followed by i.v. injection of wild-type bone marrow 24 hours after irradiation. Eight weeks after wild-type bone marrow grafting, TIE2-GFP recipient mice were exposed to 27-Gy colorectal irradiation. Tissues were collected 7 days after exposure to look for GFP-positive cells.

\section{Real-Time Quantitative PCR}

Mouse colorectal tissues were frozen in RNAlater RNA stabilization reagent (Qiagen, Valencia, CA) until analysis. Tissue and cell total RNA was prepared with the total RNA isolation kit RNeasy Mini Kit (Qiagen). After quantification on a NanoDrop ND-1000 apparatus (NanoDrop Technologies, Rockland, DE), reverse transcription was performed with $1 \mu \mathrm{g}$ RNA using a reverse transcription kit from Applied Biosystems (Courtaboeuf, France). Real-time quantitative PCR was performed on a 7900HT Fast-Real Time PCR system (Applied Biosystems) using predeveloped TaqMan Gene Expression assays (Applied Biosystems), with glyceraldehyde-3-phosphate dehydrogenase for cells and 18S for tissues as housekeeping genes. Relative mRNA was quantified using the $\Delta \Delta C_{\mathrm{T}}$ method. DataAssist software version 3.01 (Life Technologies) was used to perform hierarchical clustering with global normalization and Pearson's correlation.

\section{Western Blot Procedures}

Cell total protein was extracted using radioimmunoprecipitation assay buffer supplemented with phosphatase and protease inhibitors (Roche Diagnostics, Meylan, France). Protein concentration was determined using a BCA assay (Sigma-Aldrich, St. Louis, MO), and equal amounts of protein were resolved by SDS-PAGE. The blocked membrane was incubated with

Figure 2 Irradiated human intestinal microvascular endothelial cells (HIMECs) show modifications in gene expression pattern resembling endothelial-to-mesenchymal transition in vitro. Assay heat map illustrating differentially expressed genes among 46 genes measured 7 days after exposure of HIMECs to 0-, 10-, or 20-Gy irradiation. For one gene considered, red represents high expression, and green represents low expression. Blue arrows indicate decreased expression of endothelial markers in irradiated HIMECs compared with controls, and orange arrows indicate increased expression of markers of radiation-induced endothelial activation and mesenchymal markers. Data are representative of three independent experiments performed in triplicate. 
A
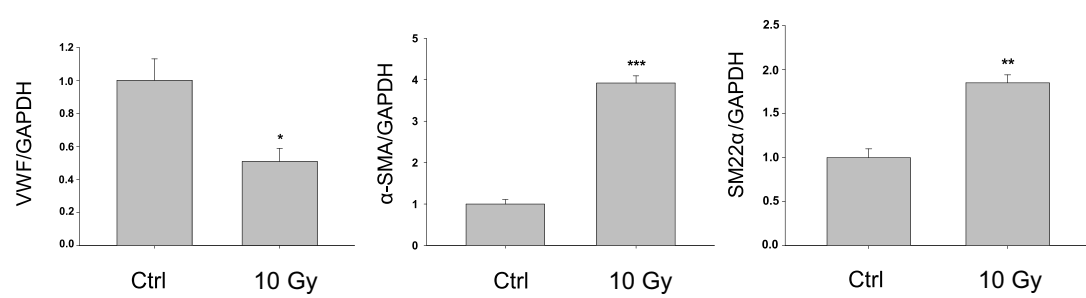

B
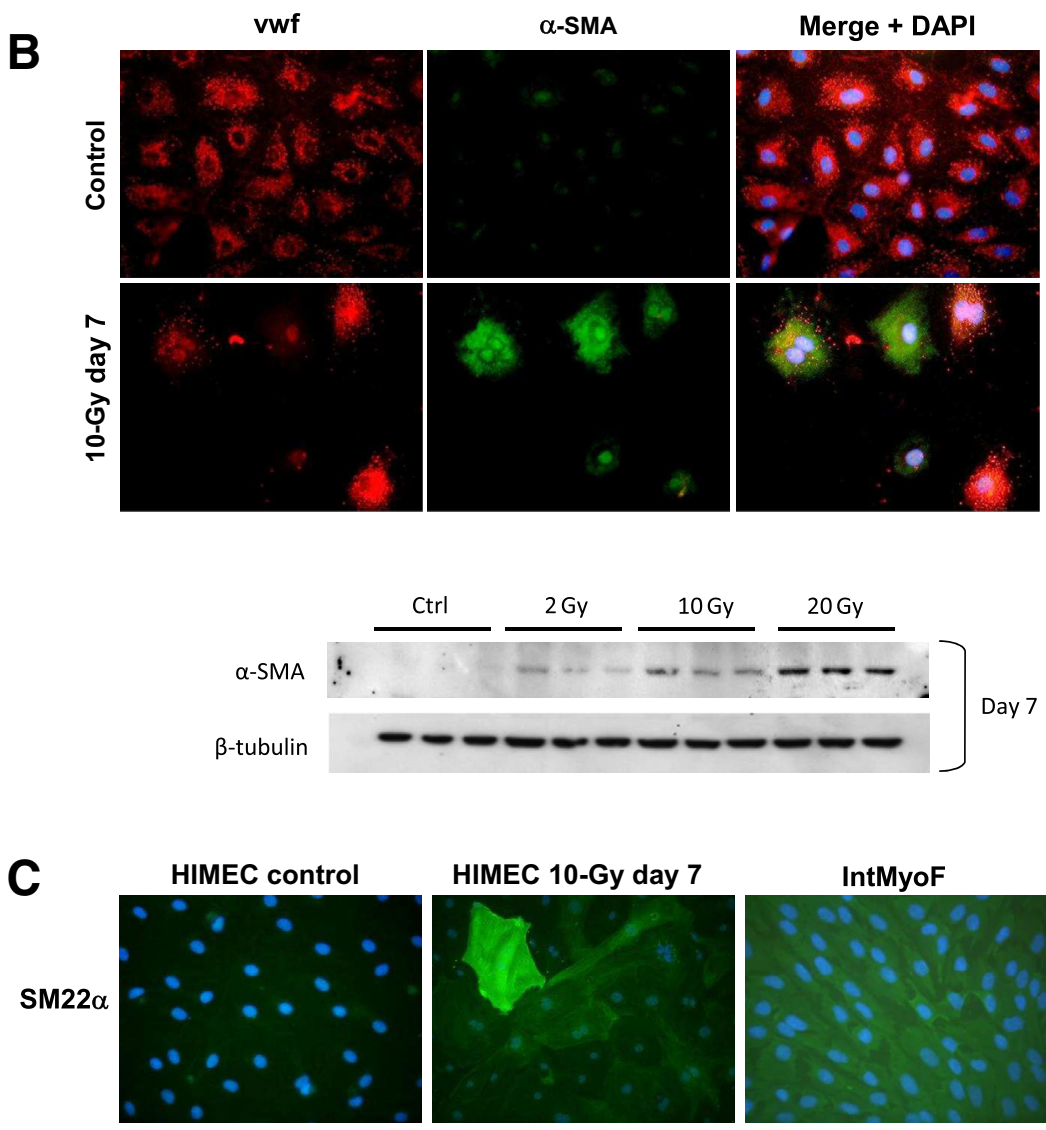

HIMEC $10-$ Gy day 7

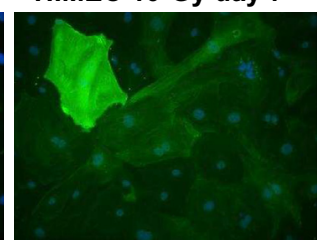

IntMyoF

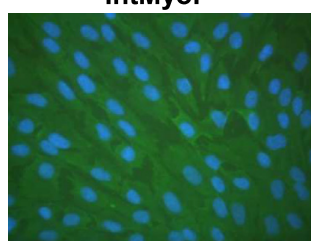

Figure 3 Irradiated human intestinal microvascular endothelial cells (HIMECs) show a mesenchymal-like phenotype in vitro. A: Real-time quantitative PCR analyses showing decreased von Willebrand factor (VWF) and increased $\alpha$-smooth muscle actin ( $\alpha$-SMA) and transgelin (smooth muscle $22 \alpha$ ) expression in 10-Gy-irradiated HIMECs compared with controls (Ctrl) 7 days after exposure. Data are representative of three independent experiments performed in triplicate. B: Immunofluorescence images showing double labeling of VWF and $\alpha$-SMA in control and 10-Gy-irradiated HIMECS 7 days after exposure, highlighting phenotypic changes observed during the endothelial-tomesenchymal transition (EndoMT) process. Increased $\alpha$-SMA mRNA expression was confirmed by Western blot analysis at day 7 for 2, 10, and $20 \mathrm{~Gy}$. C: Immunofluorescence images illustrating the radiation-induced EndoMT process with de novo expression of smooth muscle $22 \alpha$ in 10-Gyirradiated HIMECs 7 days after exposure, compared with constitutive expression in intestinal myofibroblasts (IntMyoFs). Nuclei were counterstained with DAPI. ${ }^{*} P<0.05$, ${ }^{* *} P<0.01$, and ${ }^{* * *} P<0.001$. Original magnification, $\times 400$ (B and C). GAPDH, glyceraldehyde-3-phosphate dehydrogenase. primary antibodies overnight at $4^{\circ} \mathrm{C}$. Bands were detected by using a horseradish peroxidase secondary antibody and visualized with ECL Plus reagent (GE Healthcare, Buc, France). $\beta$ Tubulin or glyceraldehyde-3-phosphate dehydrogenase was used as loading control (Santa Cruz Biotechnology).

\section{Statistical Analysis}

Data are given as means \pm SEM. Statistical analyses were performed by analysis of variance, $t$-test, or Mann-Whitney rank sum test when appropriate, with a level of significance of $P<0.05$.

\section{Results}

Irradiation Modulates HIMECs to a Mesenchymal-Like Phenotype in Vitro via TGF/Smad Pathway Activation

The purity and endothelial nature of HIMECs were verified by repeated flow cytometric sorting, real-time quantitative PCR, immunostaining, and the Dil-Ac-LDL (Cayman Chemicals, Ann Arbor, MI) incorporation test until 20 passages, as described by Rieder et al. ${ }^{20}$ HIMECs in the present study were used between passages 11 and 15 .

To induce EndoMT, confluent HIMECs were exposed to single doses of 0-, 2-, 10-, and 20-Gy irradiation and monitored until day 21 after exposure. Irradiated HIMECs acquired some features of radiation-induced endothelial cell activation observed in other cell lines, such as increased expression of plasminogen activator inhibitor-type 1, tissue factor, and Eselectin (Figure 1A), together with a radiation-induced global increase in stress fibers, as illustrated by phalloidin staining (Figure 1B). Irradiated HIMECs also showed gene expression modifications compatible with EndoMT. Heat map representation with 46 genes measured 7 days after exposure of HIMECs to 0,10 , or 20 Gy discriminated between unirradiated cells and 10- or 20-Gy-irradiated cells (Figure 2). Radiation exposure decreases expression by HIMECs of endothelial cell markers, such as vascular endothelial 
cadherin (CDH5), CD31 (platelet endothelial cell adhesion molecule), vWF, $\beta$-catenin (CTNNB1), Tie1, and Tie2. Decreased expression of endothelial cell markers occurs with a concurrent increase in actor mediators of EndoMT, such as HEY1 and HEY2 (Figure 2), and in mesenchymal cell markers, such as $\alpha$-SMA (ACTA2) and smooth muscle $22 \alpha$ (Figures 2 and 3A). Immunofluorescence double staining of vWF and $\alpha$-SMA in control and irradiated HIMECs showed that within the irradiated cell population, some cells lose vWF and concurrently show increased $\alpha$-SMA immunoreactivity, whereas other cells maintain vWF immunoreactivity (Figure 3B). This may suggest that radiation-induced EndoMT does not affect all irradiated HIMECs or that phenotypic changes may occur at different time points, at least in vitro. Increased $\alpha$-SMA expression was confirmed at the protein level by Western blot analysis and is shown for the day 7 time point (Figure 3B). Radiation-induced EndoMT is also illustrated by immunofluorescence staining for smooth muscle $22 \alpha$. Smooth muscle $22 \alpha$ immunoreactivity was absent in control HIMECs, whereas there was a global increase in the entire irradiated cell population, with some cells showing strongly positive cable-shaped immunoreactivity (Figure 3C).

Transformed HIMECs showed no change in Smad3 mRNA and protein levels (Figure 4, A and B), but Western blot and immunostaining studies revealed that irradiated HIMECs have increased phospho-Smad2/3 protein levels (Figure 4, B and C), suggesting activation of the canonical TGF/Smad pathway during the EndoMT process.

The conversion of endothelial cells into mesenchymal cells may involve the passage through a multipotent stemlike phenotype, as demonstrated by Medici et al. ${ }^{7} \mathrm{We}$, therefore, measured the expression of several hematopoietic stem and progenitor cells (CD34, vascular endothelial growth factor receptor 2, and CD133) and stem cell markers (CD10, CD44, CD71, CD90, and CD117). CD133, CD10, and CD90 expression was not detected under the mentioned conditions. Only CD34 expression significantly increased after radiation exposure (Supplemental Figure S1).

\section{EndoMT Occurs in Human Radiation Proctitis}

EndoMT has been reported in colonic Crohn disease and ulcerative colitis human tissues, in mucosal vessels in close proximity to inflammatory infiltrates. EndoMT was visualized by immunofluorescence and double labeling of both an endothelial marker (vWF) and a mesenchymal marker $(\alpha-$ SMA). ${ }^{20}$ At 5 to 7 weeks after the end of radiation therapy, radiation proctitis is characterized by mucosal and submucosal inflammation and collagen deposition, ${ }^{25,29}$ a pathological context known to favor EndoMT. Moreover, a previous study showed increased endothelial phosphoSmad2/3 immunostaining in cases of human radiation proctitis, ${ }^{25}$ suggesting radiation-induced activation of the canonical TGF/Smad pathway recognized as an EndoMT inducer. Colocalization of vWF and $\alpha$-SMA was observed in mucosal and submucosal vessels of human radiation proctitis, but not in normal rectal tissue (Figure 5A), suggesting that EndoMT may participate in tissue damage after radiation exposure. Figure 5B shows the percentage of patients showing EndoMT according to tissue radiation injury score (Supplemental Table S1). To further explore EndoMT in vivo, we used a preclinical model of radiation proctitis in transgenic mice.

\section{Evidence for EndoMT in Vivo in a Preclinical Model of Radiation Proctitis}

EndoMT has been implicated in several models of tissue injury with common features of inflammation and fibrosis. The preclinical model of radiation proctitis used herein was characterized by severe acute inflammation progressing to subsequent tissue fibrosis, pathophysiological processes that may favor EndoMT. To monitor eventual radiation-induced loss of endogenous endothelial markers and allow the recognition of mesenchymal marker-expressing cells of endothelial origin, radiation proctitis was induced in endothelial reporter mice expressing GFP under the control of the endothelial-specific promoter Tie2. As previously described, ${ }^{29}$ acute radiation proctitis (day 14) consisted of extensive mucosal ulceration with complete loss of crypt architecture and dense transmural inflammation (Figure 6). Late damage (14 weeks) was characterized by dystrophic mucosal regeneration with glandular atypia, including colitis cystica profunda, and matrix deposition, especially in the mucosal and submucosal compartments. The development of radiation proctitis was associated with a progressive
A

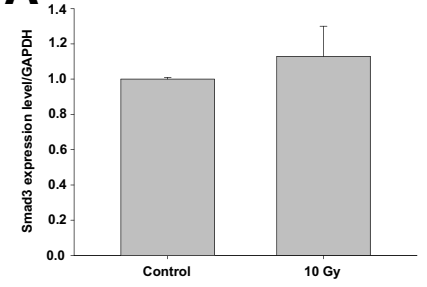

B

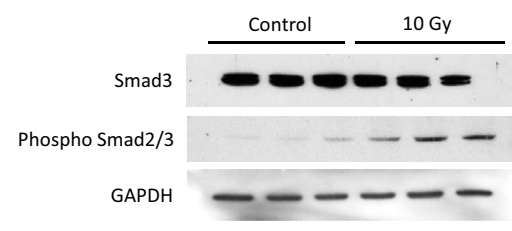

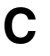

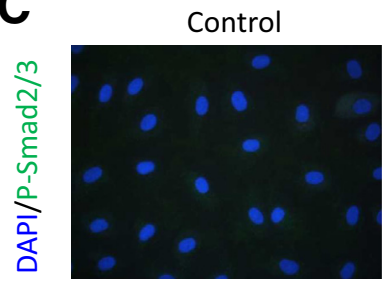

$10 \mathrm{~Gy}$

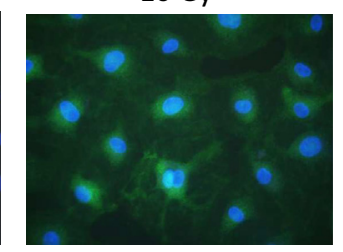

Figure 4 Smad3 activation in human intestinal microvascular endothelial cells (HIMECs) 7 days after 10-Gy radiation exposure. A: HIMEC irradiation does not change Smad3 mRNA levels. B: Smad3 protein levels are unchanged after radiation exposure, but phospho-Smad2/3 increases. C: Radiation-induced increase in phospho-Smad2/3 protein levels are confirmed by immunofluorescence staining using anti-phospho-Smad2/3 antibody. Data are representative of three independent experiments. GAPDH, glyceraldehyde-3-phosphate dehydrogenase. 
A

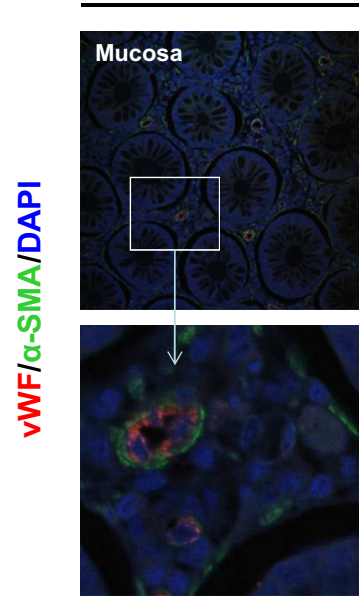

IR

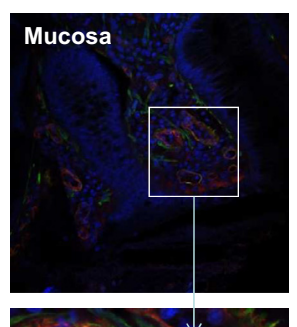

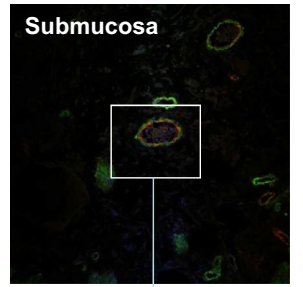

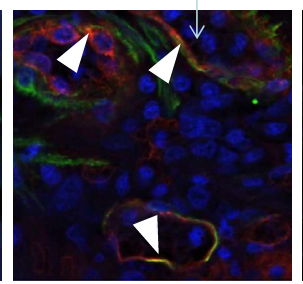

B

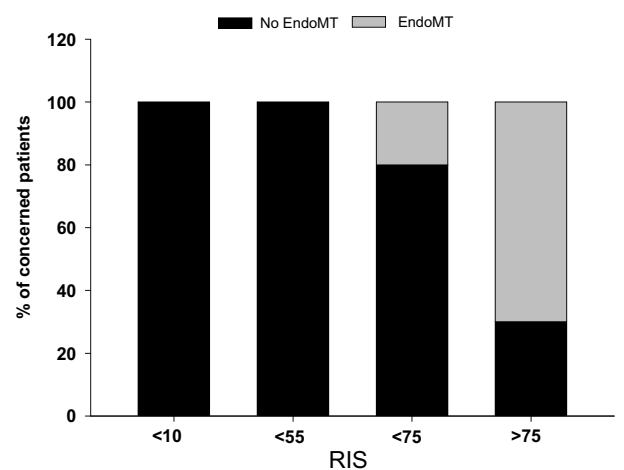

Figure 5 Endothelial-to-mesenchymal transition (EndoMT) is present in irradiated (IR) human rectum. A: Immunofluorescence double labeling of von Willebrand factor (vWF; red) and $\alpha$-smooth muscle actin ( $\alpha$-SMA; green) in human rectal tissues 5 to 7 weeks after 45 -Gy preoperative radiation therapy for rectal adenocarcinoma. Co-expression of vWF and $\alpha$-SMA (yellow) is observed in the chorionic mucosal microvessels and in the submucosal vessels of pathological tissues, but not in control (CTRL) tissues. Enlargements of boxed areas show co-expression indicated with arrowheads. Nuclei were counterstained with DAPI. B: Percentage of patients concerned with EndoMT according to the radiation injury score (RIS). $n=4$ (RIS, $<10$ and $<55$ ); $n=5$ (RIS, $<75$ ); $n=11$ (RIS, $>75$ ). Original magnification, $\times 200$ (A).

increase in $\alpha$-SMA immunoreactivity (Figure 6 ). $\alpha$-SMA is expressed by smooth muscle cells, transiently expressed by fibroblast-derived myofibroblasts in wound healing conditions, and sustainably expressed in fibroproliferative diseases. ${ }^{30,31}$ Thus, we investigated whether cells of endothelial origin (GFP positive) contribute to global $\alpha$-SMA expression in pathological tissue.

Radiation-induced mucosal damage was characterized by substantial infiltration of immune cells and by a dense microvascular network. Tie2 is considered as endothelial specific, but is also known to be expressed by a subset of myeloid cells. ${ }^{32}$ The co-immunostaining of inflamed tissues with both anti-GFP antibody (for endothelial cells) and $\alpha$ SMA with corresponding fluorescent secondary antibodies (green for GFP and red for $\alpha$-SMA) showed numerous GFPpositive cells outside the vascular endothelium, which were difficult to identify as myeloid cells or capillary endothelial cells (Supplemental Figure S2A). To see if eventual Tie2positive myeloid cells represent an obstacle to the assessment of GFP/ $\alpha$-SMA staining, we grafted wild-type bone marrow in Tie2-GFP recipient mice to turn off bone marrow expression of Tie2 (and GFP). Tie2-GFP mice showed a pan-endothelial staining pattern of GFP lining the internal part of the blood vessels with sparse round GFP-positive cells mainly located in the mucosa, suggestive of immune cells (Supplemental Figure S2B). Acute inflammatory infiltrate in these mice showed a negligible participation of these round-shaped cells in the global radiation-induced infiltrate (Supplemental Figure S2B). Wild-type bone marrow grafting in Tie2-GFP mice did not change the staining pattern in control or in irradiated tissues, suggesting that GFP-positive immune cells may not compromise the monitoring of GFP-positive endothelial cells during the development of tissue radiation damage.

To monitor the EndoMT process, endothelial cells expressing the mesenchymal marker $\alpha$-SMA were tracked
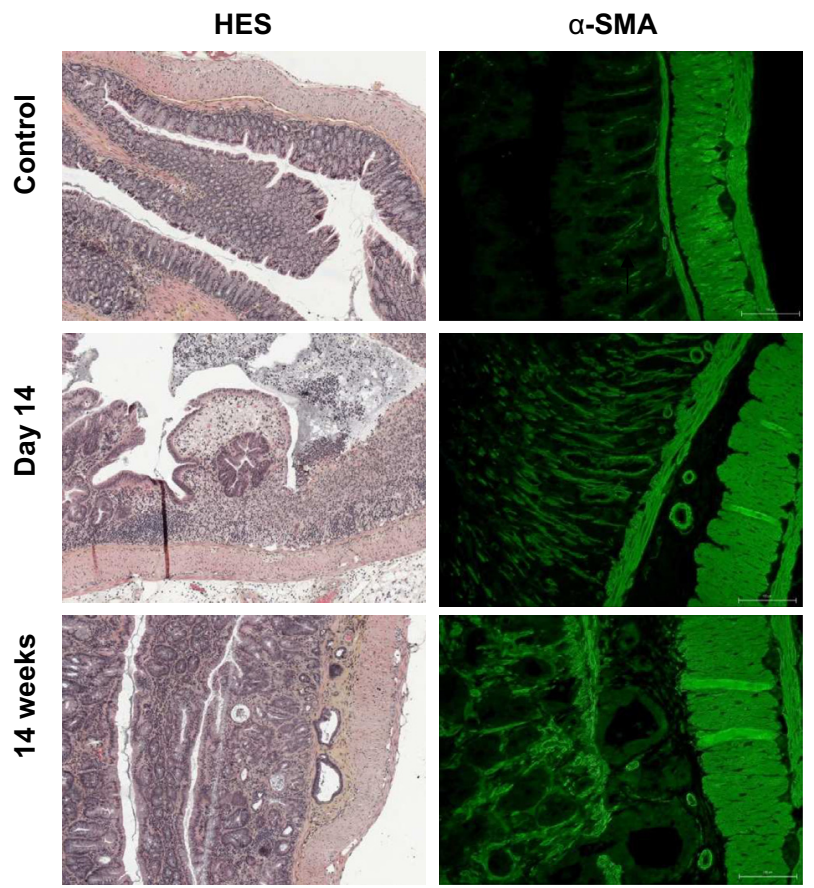

Figure 6 Radiation proctitis in mice is associated with increased $\alpha$-smooth muscle actin ( $\alpha$-SMA) immunoreactivity. Images of hemalun-eosin-safran (HES) and $\alpha$-SMA immunofluorescence staining of control and 27-Gy-irradiated tissues during the inflammatory (day 14) and fibrotic (14 weeks) phases showing severe acute transmural inflammation/mucosal ulceration and late mucosal atypia/transmural collagen deposition. Tissue damage progression is associated with a gradual increase in $\alpha$-SMA immunoreactivity. Original magnification, $\times 100$ (left panels); $\times 200$ (right panels). 


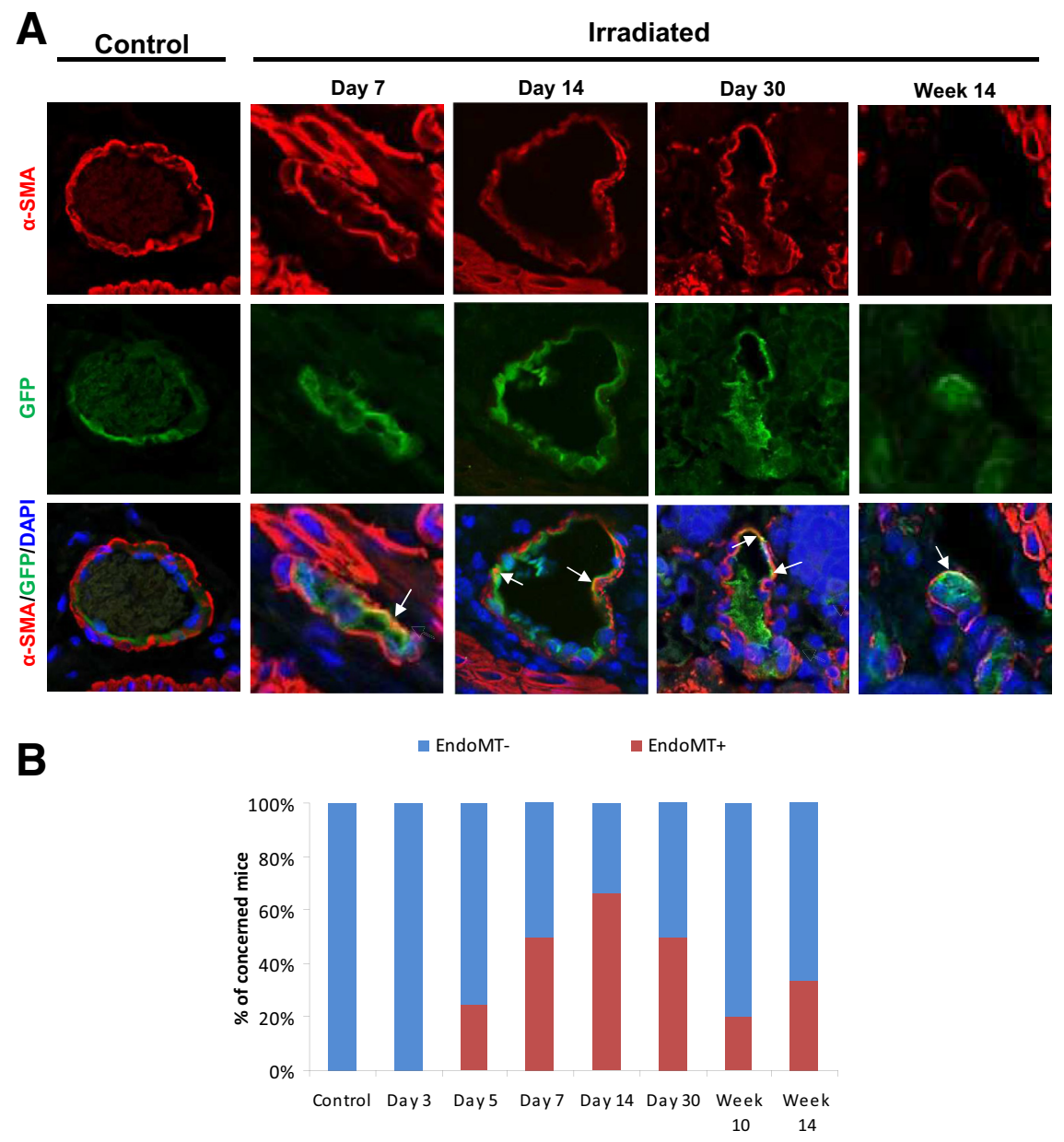

Figure 7 In vivo evidence of endothelial-tomesenchymal transition (EndoMT) in the preclinical mouse model of radiation proctitis in mice expressing the green fluorescent protein (GFP) under the control of the endothelial promoter Tie2. A: Representative images of confocal microscopy of immunofluorescence double labeling of $\alpha$-smooth muscle actin ( $\alpha$-SMA; mesenchymal marker, red) and GFP (endothelial marker, green) in control and 27-Gy-irradiated mouse rectum 7, 14, and 30 days and 14 weeks after exposure. $\alpha$-SMA ${ }^{+} /$GFP $^{+}$ double-labeled cells (yellow, arrows) are observed in small vessels of the mucosa and submucosa of irradiated tissues whatever the time after irradiation. No double labeling was observed in control tissues. B: Percentage of control and 27-Gyirradiated Tie2-GFP mice concerned with EndoMT from 3 days to 14 weeks after exposure. $n=3$ (control); $n=5$ (irradiated animals). Original magnification, $\times 400($ A) .

by double-immunofluorescence staining in control and irradiated tissues. Double-stained cells showed a yellow fluorescent signal, indicative of EndoMT. Images of GFP/ $\alpha$-SMA co-expressing cells were noted at different times after exposure $(7,14$, and 30 days or 14 weeks after irradiation), with higher frequency during the inflammatory phase (Figure 7). No double-stained cells were observed in corresponding control tissues. Co-expression of mesenchymal and endothelial markers has been demonstrated in the vascular endothelium, but also within fibrotic areas in different models of tissue damage. In our case, no signal was observed outside the endothelial lining of vessels. We assumed that Tie2 (and GFP) expression may be shut off on cell differentiation in vivo and may prevent the fluorescent signal, especially given the strong mesenchymal marker fluorescence intensity. GFPpositive cells were thus sought outside the endothelial compartment in areas of fibrosis by GFP immunostaining alone, and serial section staining with Sirius red to identify collagen deposition. Staining revealed the presence of elongated GFP-positive cells in areas of mucosal and submucosal fibrosis (Figure 8A), suggesting mesenchymal cells of endothelial origin and confirming the EndoMT process as a potential participant in the development of rectal radiation fibrosis. No such GFPpositive elongated cells were observed in control tissues (Figure 8B).

\section{EndoMT in Vivo Occurs Together with the Canonical TGF- $\beta /$ Smad Pathway Activation}

In our preclinical model of radiation proctitis, rectal inflammation 14 days after radiation exposure was characterized by changes in mRNA expression levels of several factors recognized as EndoMT participants (Figure 9A). For example, inflammatory mediators, such as IL-1 $\beta$ and tumor necrosis factor- $\alpha$, are known to induce HIMEC EndoMT in vitro, and Snail and Zeb are EndoMT transcription factors. Moreover, irradiated tissues show increased expression of TGF- $\beta$, able to induce EndoMT in vitro, and some TGF- $\beta$ target genes, such as SERPINE1 (plasminogen activator inhibitor-type 1), $M M P 2$, and $M M P 9$. Irradiation also increases tissue expression of mesenchymal markers, such as $\alpha$-SMA, Col3A1, or smooth muscle $22 \alpha$, suggesting TGF/Smad pathway activation in vivo. To confirm TGF/Smad activation, fluorescent co-immunostainings of GFP and phospho-Smad2/3 were performed on irradiated tissues (Figure 9B). Images showed that radiation 


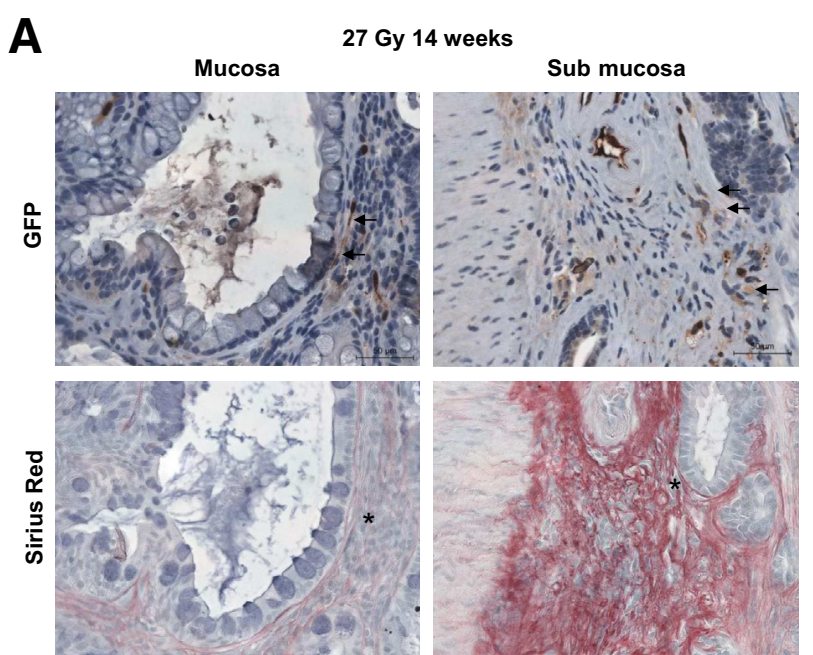

B

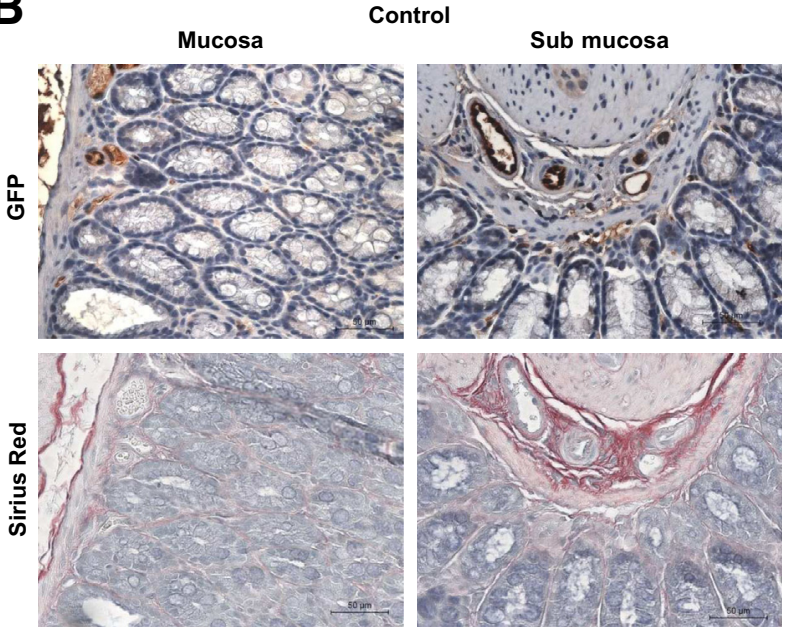

Figure 8 Presence of elongated green fluorescent protein (GFP) ${ }^{+}$cells outside the vascular endothelium. A: GFP immunostaining and Sirius red staining in 27-Gy-irradiated tissues showing mucosal and submucosal compartments. Elongated $\mathrm{GFP}^{+}$cells (arrows) are seen around dystrophic crypts in the mucosa in areas of collagen deposition revealed by Sirius red staining (asterisk). In the submucosa, areas of dense Sirius red staining (asterisk) show GFP ${ }^{+}$cells outside the vascular compartment, suggesting an endothelial origin (arrows). B: No elongated $\mathrm{GFP}^{+}$cells are observed in control tissues. Original magnification, $\times 400(\mathbf{A}$ and $\mathbf{B})$.

exposure induced phospho-Smad2/3 immunoreactivity in numerous cells' nucleus, including endothelial cells. Data suggest canonical TGF/Smad pathway activation 14 days after radiation exposure, the time at which we observed a higher frequency of EndoMT.

\section{Discussion}

It is now well established that endothelial cells are the cornerstone of the initiation and development of radiation damage to healthy normal tissues. ${ }^{11,12}$ Radiation-induced endothelial cell apoptosis and a long-lasting activated phenotype both favor vascular leakage, microvascular thrombosis, and immune cell recruitment. $^{11}$
In this study, we demonstrated that irradiated HIMECs derived from normal human colonic mucosa show increased expression of endothelial cell activation markers, such as tissue factor, plasminogen activator inhibitor-type 1, or Eselectin, and increased stress fiber formation. Otterson et $\mathrm{al}^{33}$ showed that irradiation of HIMECs induced cell apoptosis and stress fiber formation, decreased cell migration in a scratch injury model in vitro, and reduced in vitro tube-like structure formation. Protection from radiation effects by a superoxide dismutase mimetic reveals that irradiated HIMECs are subject to an oxidant microenvironment. ${ }^{33}$ These features of endothelial cell oxidative stress, apoptosis, and global functional modifications after irradiation have been demonstrated in more common macrovascular and microvascular cell lines after radiation exposure..$^{12,34,35}$ The innovative concept in the present study was to determine whether radiation-induced phenotypic changes in HIMECs resemble EndoMT and whether this process participates in radiation-induced intestinal fibrosis. Several published findings were in favor of the possibility of inducing EndoMT by irradiating HIMECs. First, mediators of inflammation, TGF- $\beta$ signaling, and oxidative stress, all features of cell response to radiation exposure, induce EndoMT in different cell lines. ${ }^{21-23}$ Second, HIMECs undergo EndoMT when exposed to recombinant cytokines or ex vivo inflammatory mediators. ${ }^{20}$ Frid et al ${ }^{36}$ demonstrated, for the first time, that, as embryonic vasculature, adult bovine endothelial cells can acquire a smooth muscle phenotype in vitro. Interestingly, they used cell irradiation to stop cell proliferation. They thus demonstrated that EndoMT was independent of cell proliferation and still occurred in quiescent irradiated endothelial cells. Even though the authors did not specify whether irradiation increased the number of transdifferentiating cells, this was the first study to report EndoMT after irradiation. Kim et $\mathrm{al}^{37}$ have shown that irradiation induces macrovascular endothelial cell transformation into a mesenchymal phenotype in vitro and that a proportion of the mesenchymal cells found in atherosclerotic tissues of irradiated apoprotein E-null mice were of endothelial origin. Radiation-induced EndoMT was confirmed in HIMECs in the present study. Irradiated HIMECs reduced their expression of several endothelial markers and showed de novo expression of mesenchymal markers, the hallmark of EndoMT. Irradiated HIMECs also showed reduced Tie1 expression, a deficiency that induces human dermal microvascular endothelial cells to undergo EndoMT. ${ }^{11}$ Moreover, we showed that EndoMT was associated with increased phospho-Smad $2 / 3$ protein levels, suggesting involvement of the canonical TGF/Smad pathway in radiation-induced EndoMT in vitro in our model. This was already demonstrated in other experimental conditions inducing EndoMT but also in models of epithelialto-mesenchymal transition. ${ }^{6,23,38}$

The conversion of endothelial cells into mesenchymal cells may involve the passage through a multipotent stem-like 

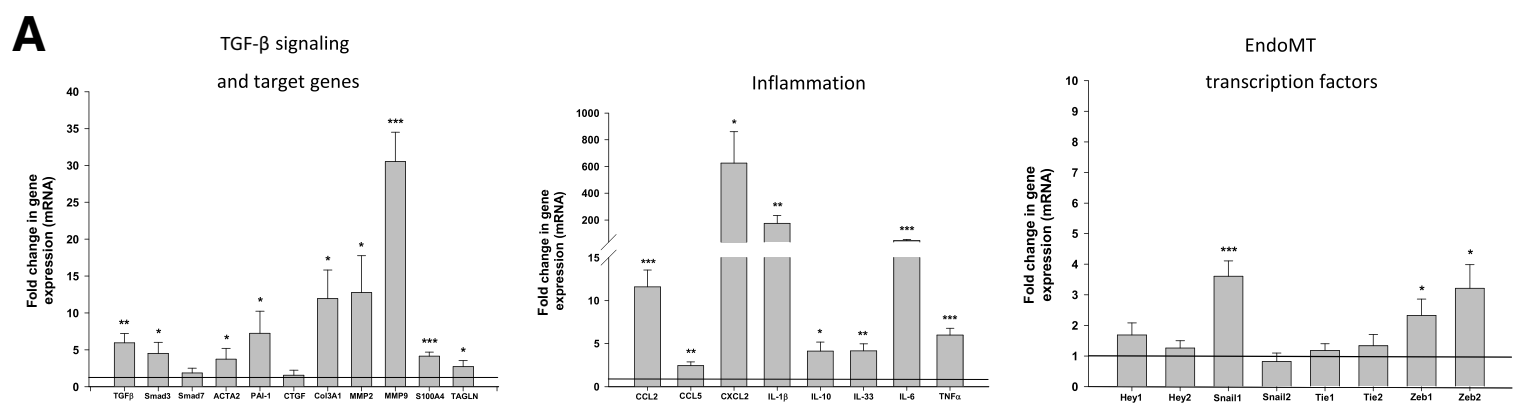

B

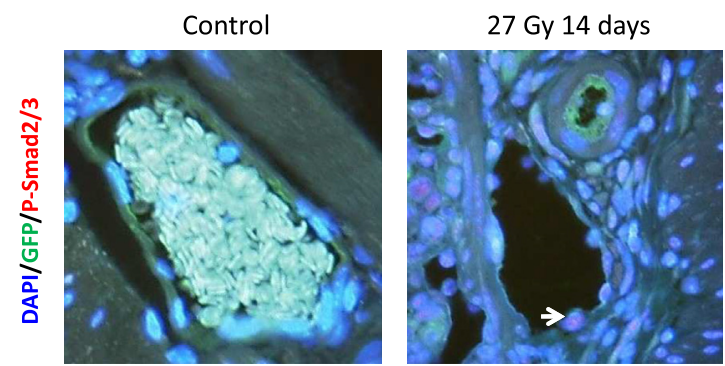

Figure 9 Endothelial-to-mesenchymal transition (EndoMT) in vivo occurs together with the canonical TGF/Smad pathway activation. A: Fold changes in gene expression in 27-Gy-irradiated rectal tissues 14 days after exposure, the time at which the higher frequency of EndoMT was observed. PCR analyses showed an increase in TGF- $\beta 1$, Smad 3, and TGF- $\beta$ target genes, several inflammatory mediators known as EndoMT inducers [IL-1 $\beta$ and tumor necrosis factor (TNF)- $\alpha$ ], and EndoMT-related transcription factors. B: Immunofluorescence images of double labeling of GFP and phospho-Smad2/3 in control and $27-$ Gy-irradiated rectum, 14 days after exposure. Immunostaining shows radiation-induced nuclear localization of phospho-Smad2/3 in numerous cell types, including endothelial cells (arrow). $n=5$ (control); $n=6$ (irradiated animals). ${ }^{*} P<0.05,{ }^{*} P<0.01$, and ${ }^{* * *} P<0.001$. Original magnification, $\times 400$ (B). $\mathrm{CCL}$, chemokine ligand; CTGF, connective tissue growth factor; MMP, matrix metalloproteinase; PAI, plasminogen activator inhibitor.

phenotype. $^{7,39}$ Irradiated HIMECs did not show significant variations in expression of mesenchymal stem cell markers. Concerning hematopoietic stem cell and progenitor cell markers, only CD34 expression increased after exposure of HIMECs to radiation. CD34 is regarded as a marker of vascular endothelial progenitor cells in the peripheral blood, but has numerous other biological functions. ${ }^{40,41}$ Primary human umbilical vein endothelial cells in vitro are CD34 positive and gradually lose $\mathrm{CD} 34$ expression during passages and cell maturation. The remaining CD34-positive cells represent a subpopulation of elongated cells with specific adhesion and migration properties resembling sprouting tip cells during in vivo angiogenesis. ${ }^{42,43} \mathrm{CD} 34$ tends to be considered as the guardian of cell plasticity, and CD34 overexpression may favor EndoMT and transformed cell mobility. This remains to be studied.

HIMECs are primary isolated cells from normal human colon and thus represent a highly relevant model to investigate whether EndoMT occurs and participates in the excessive scarring process in human irradiated gut. We show, for the first time, that EndoMT exists in human rectum 5 to 7 weeks after 45-Gy preoperative radiation therapy for rectal adenocarcinoma. Transforming endothelial cells lose their endothelial markers, thus compromising observation of mesenchymal cells of endothelial origin in human irradiated tissue. To investigate the reality of radiation-induced EndoMT in vivo and to have access to multiple time points in the development of radiation-induced tissue damage, we used a model of radiation proctitis in endothelial cell reporter mice. In this model, GFP-expressing endothelial cells can be monitored throughout their transformation. ${ }^{4,20,23}$ Areas of radiation-induced rectal inflammation and fibrosis show EndoMT in the vascular endothelium of mucosal and submucosal vessels. EndoMT occurred in a proinflammatory mediators overexpression context of several mediators (IL-1 $\beta$, tumor necrosis factor- $\alpha$, and TGF- $\beta$ ) known to induce EndoMT in vitro, ${ }^{20}$ EndoMT-related transcription factors, concurrent with the overexpression of several TGF- $\beta$ target genes, and associated with phospho-Smad2/3 immunoreactivity in endothelial cells' nuclei. Data suggest that EndoMT readily exists in our preclinical model of radiationinduced proctitis and may occur via the TGF/Smad pathway activation, as demonstrated in vitro.

However, and in contrast to several studies showing mesenchymal cells of endothelial origin outside the vascular compartment in models of lung, kidney, or intestinal fibrosis, ${ }^{20,44,45}$ we did not observe signals outside the endothelial compartment after double-immunofluorescence staining. It is possible that Tie2 (and GFP) expression was shut off or even reduced on cell differentiation in vivo in our model, and became invisible given the strong fluorescent signal obtained for $\alpha$-SMA. We thus performed GFP immunostaining alone on tissue sections presenting areas of fibrosis (highlighted by Sirius red staining). We then observed some GFP-positive spindle-shaped cells in those areas, suggesting mesenchymal cells of endothelial origin 


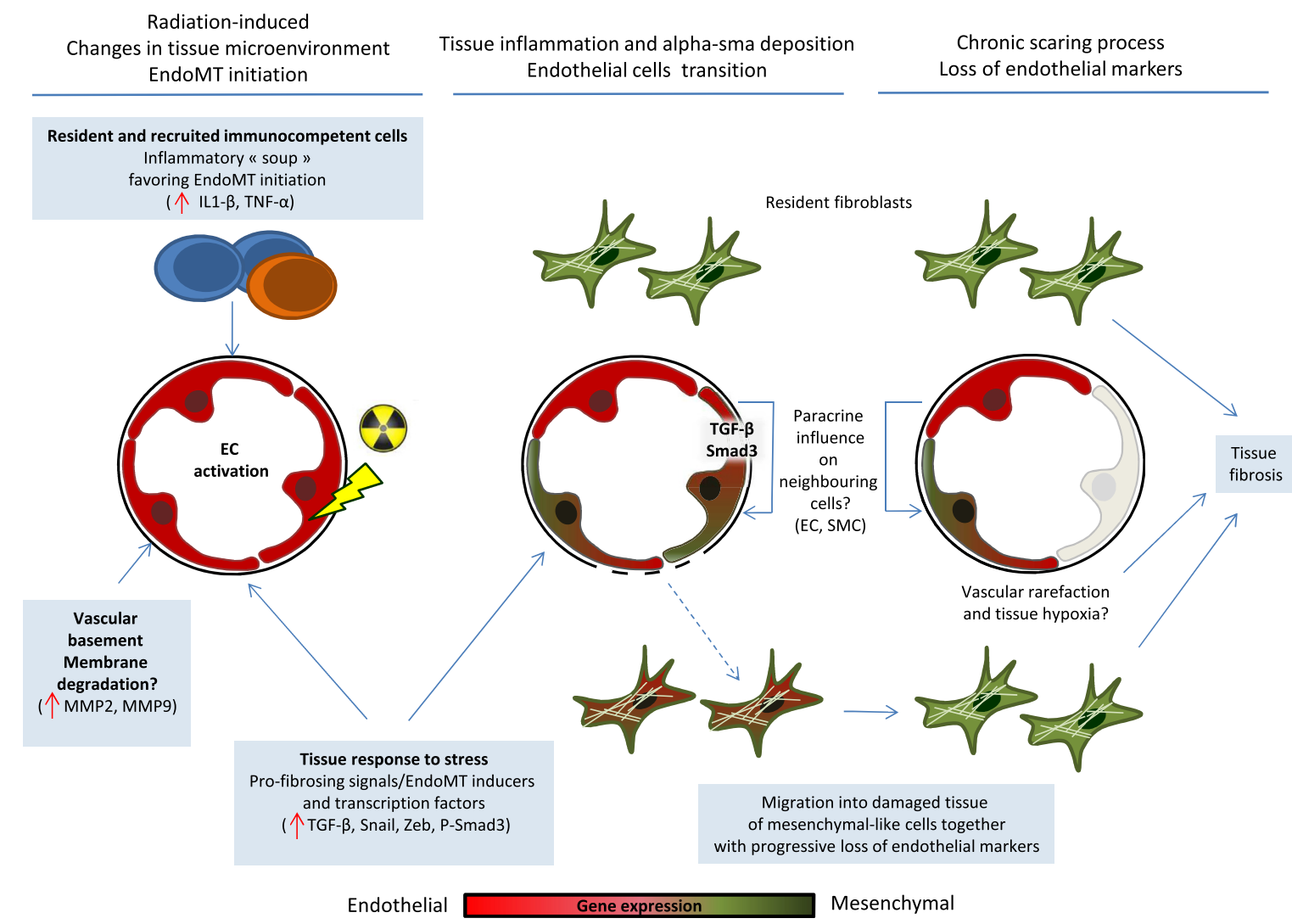

Figure 10 Radiation-induced endothelial-to-mesenchymal transition (EndoMT). Radiation-induced changes in tissue microenvironment favor EndoMT by the activation of resident cells and/or recruitment of different cell types, resulting in the overexpression of molecular factors known as EndoMT inducers or EndoMT transcription factors, or implicated in the basement membrane degradation necessary to cell movement during transition. Perduring tissue inflammation maintains tissue stress and allows endothelial cell (EC) transition via transforming growth factor (TGF)- $\beta /$ Smad pathway activation. Transformed ECs may stay in the vascular compartment and exert paracrine influence on neighboring cells. ECs can also move within the tissue while progressively losing their endothelial markers, and participate in the scarring process together with other mesenchymal cells. SMC, smooth muscle cell.

possibly participating in radiation-induced tissue fibrosis. It is, however, currently impossible to estimate the percentage of mesenchymal cells of endothelial origin, because we still do not know if transforming cells completely lose their vascular features or if they remain intermediate cells.

Tie2-GFP mice are widely used to track endothelial cells, and the Tie2 promoter is used specifically to turn off genes in the endothelial compartment. However, the specificity of Tie2 promoter may be challenged. Indeed, not only endothelial cells but also some hematopoietic stem cells, pericyte precursors (spindle-shaped cells around tumor vessels), and a fraction of tumor-infiltrating leukocytes have been identified as proangiogenic Tie2-expressing monocytes. ${ }^{46}$ Herein, highly GFP-positive cells observed outside the endothelial compartment in control tissues were sparse and round, so probably not pericytes. We turned off hematopoietic progenitor Tie2 expression by wild-type bone marrow grafting in Tie2-GFP recipient mice. This did not change the few nonendothelial GFP-positive round cells in unirradiated and in irradiated tissue, suggesting negligible participation of GFP-positive cells of bone marrow origin in radiation-induced inflammatory infiltrate. This strengthens our observations of the endothelial origin of mesenchymal-like low-GFP spindle-shaped cells in areas of collagen deposition in damaged rectum. Nevertheless, the presence of GFP-positive mesenchymal cells of endothelial origin outside the vascular endothelium seems not to be a prerequisite for EndoMT to participate in tissue fibrosis, because transformed endothelial cells may influence surrounding cells, such as vascular smooth muscle cells or pericytes, through a paracrine action and favor tissue fibrosis. ${ }^{25}$

In conclusion, we report herein that EndoMT readily occurs in cases of human radiation proctitis after external beam radiation therapy. In vivo radiation-induced EndoMT is found again in a preclinical model of radiation proctitis in mice, and in vitro on isolated human microvascular endothelial cells, both via, at least in part, the activation of the canonical TGF/ Smad pathway. Figure 10 shows a schematic representation of the events that may occur in irradiated healthy tissue and favor EndoMT, and the possible consequences of endothelial cells' phenotypic conversion. As demonstrated in more and more diseases, such as tumor progression, multiple forms of tissue inflammation or fibrosis, and even rare diseases (eg, fibrodysplasia ossificans progressiva), EndoMT may contribute to radiation-induced healthy intestinal tissue damage and may, once precise mechanisms are deciphered, represent an interesting therapeutic target in cases of pelvic radiation disease. 


\section{Supplemental Data}

Supplemental material for this article can be found at http://dx.doi.org/10.1016/j.ajpath.2015.04.028.

\section{References}

1. van Meeteren LA, ten Dijke P: Regulation of endothelial cell plasticity by TGF-beta. Cell Tissue Res 2012, 347:177-186

2. Liebner S, Cattelino A, Gallini R, Rudini N, Iurlaro M, Piccolo S, Dejana E: Beta-catenin is required for endothelial-mesenchymal transformation during heart cushion development in the mouse. J Cell Biol 2004, 166:359-367

3. Arciniegas E, Frid MG, Douglas IS, Stenmark KR: Perspectives on endothelial-to-mesenchymal transition: potential contribution to vascular remodeling in chronic pulmonary hypertension. Am J Physiol Lung Cell Mol Physiol 2007, 293:L1-L8

4. Zeisberg EM, Tarnavski O, Zeisberg M, Dorfman AL, McMullen JR, Gustafsson E, Chandraker A, Yuan X, Pu WT, Roberts AB, Neilson EG, Sayegh MH, Izumo S, Kalluri R: Endothelial-to-mesenchymal transition contributes to cardiac fibrosis. Nat Med 2007, 13 : 952-961

5. He J, Xu Y, Koya D, Kanasaki K: Role of the endothelial-tomesenchymal transition in renal fibrosis of chronic kidney disease. Clin Exp Nephrol 2013, 17:488-497

6. Cooley BC, Nevado J, Mellad J, Yang D, St Hilaire C, Negro A, Fang F, Chen G, San H, Walts AD, Schwartzbeck RL, Taylor B, Lanzer JD, Wragg A, Elagha A, Beltran LE, Berry C, Feil R, Virmani R, Ladich E, Kovacic JC, Boehm M: TGF-beta signaling mediates endothelial-to-mesenchymal transition (EndMT) during vein graft remodeling. Sci Transl Med 2014, 6:227ra234

7. Medici D, Shore EM, Lounev VY, Kaplan FS, Kalluri R, Olsen BR: Conversion of vascular endothelial cells into multipotent stem-like cells. Nat Med 2010, 16:1400-1406

8. Zeisberg EM, Potenta S, Xie L, Zeisberg M, Kalluri R: Discovery of endothelial to mesenchymal transition as a source for carcinomaassociated fibroblasts. Cancer Res 2007, 67:10123-10128

9. Denham JW, Hauer-Jensen M: The radiotherapeutic injury: a complex "wound." Radiother Oncol 2002, 63:129-145

10. Bentzen SM: Preventing or reducing late side effects of radiation therapy: radiobiology meets molecular pathology. Nat Rev Cancer 2006, 6:702-713

11. Wang J, Boerma M, Fu Q, Hauer-Jensen M: Significance of endothelial dysfunction in the pathogenesis of early and delayed radiation enteropathy. World J Gastroenterol 2007, 13:3047-3055

12. Milliat F, Francois A, Tamarat R, Benderitter M: [Role of endothelium in radiation-induced normal tissue damages] French. Ann Cardiol Angeiol (Paris) 2008, 57:139-148

13. Yarnold J, Brotons MC: Pathogenetic mechanisms in radiation fibrosis. Radiother Oncol 2010, 97:149-161

14. Hinz B, Phan SH, Thannickal VJ, Galli A, Bochaton-Piallat ML, Gabbiani G: The myofibroblast: one function, multiple origins. Am J Pathol 2007, 170:1807-1816

15. Mattoli S, Bellini A, Schmidt M: The role of a human hematopoietic mesenchymal progenitor in wound healing and fibrotic diseases and implications for therapy. Curr Stem Cell Res Ther 2009, 4:266-280

16. Kalluri R, Neilson EG: Epithelial-mesenchymal transition and its implications for fibrosis. J Clin Invest 2003, 112:1776-1784

17. Piera-Velazquez S, Li Z, Jimenez SA: Role of endothelialmesenchymal transition (EndoMT) in the pathogenesis of fibrotic disorders. Am J Pathol 2011, 179:1074-1080

18. Bataille F, Rohrmeier C, Bates R, Weber A, Rieder F, Brenmoehl J, Strauch U, Farkas S, Furst A, Hofstadter F, Scholmerich J, Herfarth H, Rogler G: Evidence for a role of epithelial mesenchymal transition during pathogenesis of fistulae in Crohn's disease. Inflamm Bowel Dis 2008, 14:1514-1527

19. Flier SN, Tanjore H, Kokkotou EG, Sugimoto H, Zeisberg M, Kalluri R: Identification of epithelial to mesenchymal transition as a novel source of fibroblasts in intestinal fibrosis. J Biol Chem 2010, 285:20202-20212

20. Rieder F, Kessler SP, West GA, Bhilocha S, de la Motte C, Sadler TM, Gopalan B, Stylianou E, Fiocchi C: Inflammation-induced endothelialto-mesenchymal transition: a novel mechanism of intestinal fibrosis. Am J Pathol 2011, 179:2660-2673

21. Medici D, Potenta S, Kalluri R: Transforming growth factor-beta2 promotes Snail-mediated endothelial-mesenchymal transition through convergence of Smad-dependent and Smad-independent signalling. Biochem J 2011, 437:515-520

22. Maleszewska M, Moonen JR, Huijkman N, van de Sluis B, Krenning G, Harmsen MC: IL-1beta and TGFbeta2 synergistically induce endothelial to mesenchymal transition in an NFkappaBdependent manner. Immunobiology 2013, 218:443-454

23. Montorfano I, Becerra A, Cerro R, Echeverria C, Saez E, Morales MG, Fernandez R, Cabello-Verrugio C, Simon F: Oxidative stress mediates the conversion of endothelial cells into myofibroblasts via a TGF-betal and TGF-beta2-dependent pathway. Lab Invest 2014, 94:1068-1082

24. Milliat F, Sabourin JC, Tarlet G, Holler V, Deutsch E, Buard V, Tamarat R, Atfi A, Benderitter M, Francois A: Essential role of plasminogen activator inhibitor type-1 in radiation enteropathy. Am J Pathol 2008, 172:691-701

25. Milliat F, Francois A, Isoir M, Deutsch E, Tamarat R, Tarlet G, Atfi A, Validire P, Bourhis J, Sabourin JC, Benderitter M: Influence of endothelial cells on vascular smooth muscle cells phenotype after irradiation: implication in radiation-induced vascular damages. Am J Pathol 2006, 169:1484-1495

26. Binion DG, West GA, Ina K, Ziats NP, Emancipator SN, Fiocchi C: Enhanced leukocyte binding by intestinal microvascular endothelial cells in inflammatory bowel disease. Gastroenterology 1997, 112:1895-1907

27. Skwarchuk MW, Travis EL: Volume effects and epithelial regeneration in irradiated mouse colorectum. Radiat Res 1998, 149:1-10

28. Skwarchuk MW, Travis EL: Changes in histology and fibrogenic cytokines in irradiated colorectum of two murine strains. Int J Radiat Oncol Biol Phys 1998, 42:169-178

29. Blirando K, Milliat F, Martelly I, Sabourin JC, Benderitter M, Francois A: Mast cells are an essential component of human radiation proctitis and contribute to experimental colorectal damage in mice. Am J Pathol 2011, 178:640-651

30. Darby I, Skalli O, Gabbiani G: Alpha-smooth muscle actin is transiently expressed by myofibroblasts during experimental wound healing. Lab Invest 1990, 63:21-29

31. Nedelec B, Ghahary A, Scott PG, Tredget EE: Control of wound contraction: basic and clinical features. Hand Clin 2000, 16:289-302

32. De Palma M, Venneri MA, Roca C, Naldini L: Targeting exogenous genes to tumor angiogenesis by transplantation of genetically modified hematopoietic stem cells. Nat Med 2003, 9:789-795

33. Otterson MF, Nie L, Schmidt JL, Link BJ, Jovanovic N, Lyros O, Rafiee P: EUK-207 protects human intestinal microvascular endothelial cells (HIMEC) against irradiation-induced apoptosis through the Bcl2 pathway. Life Sci 2012, 91:771-782

34. Blirando K, Hneino M, Martelly I, Benderitter M, Milliat F, Francois A: Mast cells and ionizing radiation induce a synergistic expression of inflammatory genes in endothelial cells by a mechanism involving p38alpha MAP kinase and (p65) NF-kappaB activation. Radiat Res 2012, 178:556-567

35. Soloviev AI, Tishkin SM, Parshikov AV, Ivanova IV, Goncharov EV, Gurney AM: Mechanisms of endothelial dysfunction after ionized radiation: selective impairment of the nitric oxide component of endotheliumdependent vasodilation. Br J Pharmacol 2003, 138:837-844

36. Frid MG, Kale VA, Stenmark KR: Mature vascular endothelium can give rise to smooth muscle cells via endothelial-mesenchymal transdifferentiation: in vitro analysis. Circ Res 2002, 90:1189-1196 
37. Kim M, Choi SH, Jin YB, Lee HJ, Ji YH, Kim J, Lee YS, Lee YJ: The effect of oxidized low-density lipoprotein (ox-LDL) on radiationinduced endothelial-to-mesenchymal transition. Int J Radiat Biol 2013, 89:356-363

38. Corallino S, Malabarba MG, Zobel M, Di Fiore PP, Scita G: Epithelialto-mesenchymal plasticity harnesses endocytic circuitries. Front Oncol 2015, 5:45

39. Medici D, Kalluri R: Endothelial-mesenchymal transition and its contribution to the emergence of stem cell phenotype. Semin Cancer Biol 2012, 22:379-384

40. Sidney LE, Branch MJ, Dunphy SE, Dua HS, Hopkinson A: Concise review: evidence for $\mathrm{CD} 34$ as a common marker for diverse progenitors. Stem Cells 2014, 32:1380-1389

41. Nielsen JS, McNagny KM: Novel functions of the CD34 family. J Cell Sci 2008, 121:3683-3692

42. Siemerink MJ, Klaassen I, Vogels IM, Griffioen AW, Van Noorden CJ, Schlingemann RO: CD34 marks angiogenic tip cells in human vascular endothelial cell cultures. Angiogenesis 2012, 15: $151-163$

43. Delia D, Lampugnani MG, Resnati M, Dejana E, Aiello A, Fontanella E, Soligo D, Pierotti MA, Greaves MF: CD34 expression is regulated reciprocally with adhesion molecules in vascular endothelial cells in vitro. Blood 1993, 81:1001-1008

44. Hashimoto N, Phan SH, Imaizumi K, Matsuo M, Nakashima H, Kawabe T, Shimokata K, Hasegawa Y: Endothelial-mesenchymal transition in bleomycin-induced pulmonary fibrosis. Am J Respir Cell Mol Biol 2010, 43:161-172

45. LeBleu VS, Taduri G, O’Connell J, Teng Y, Cooke VG, Woda C, Sugimoto H, Kalluri R: Origin and function of myofibroblasts in kidney fibrosis. Nat Med 2013, 19:1047-1053

46. De Palma M, Venneri MA, Galli R, Sergi Sergi L, Politi LS, Sampaolesi M, Naldini L: Tie2 identifies a hematopoietic lineage of proangiogenic monocytes required for tumor vessel formation and a mesenchymal population of pericyte progenitors. Cancer Cell 2005, 8:211-226 